the quadrant of the cornea lying over the foreign body (Fig. 7) and with the point of the keratome driven towards the foreign body. If the point of the keratome seems to drive too near the angle of the chamber the wound may be enlarged by side-toside movements instead of by an onward thrust. The foreign body may now be easily seized with toothless iris forceps and withdrawn. The risk of prolapse of iris is also avoided, as with corneal incisions in this situation the iris does not prolapse as is so often the case with incisions at the limbus.

In a case in which an eyelash was lying on the lens and no part lying on the iris a similar procedure was adopted, but the lash was first disengaged and gently coaxed over the iris by a stream of saline solution through the silver nozzle of a M'Keown's irrigator devised for the treatment of immature cataract.

\title{
ON A SMALL IMPROVEMENT IN SKIN GRAFTING BY
}

\section{ERNEST E. MADDOX}

BOURNEMOUTH.

I find the cutting of Thiersch grafts to be facilitated by first painting the skin (after the usual preparation) with a stiffening agent, such as a thin even layer of flexible collodion.

This stiffens the epidermis, so that it moves over the knife with less crumpling, and it also makes the application of the graft much easier in certain difficult situations.

I would not, however, advise the use of a stiffening agent in all cases, since sometimes the very softness of the graft enables it the better to fill in the inequalities of an uneven surface.

Paraffin wax could be substituted for collodion when the latter is not obtainable, but although it would help the cutting, there is the danger of fragments becoming detached and getting in the way during the application of the graft. For this reason collodion or formalized gelatine is better.

\section{ARTIFICIAL EPISTAXIS \\ BY \\ ERnEst E. MADDOX \\ BOURNEMOUTH.}

A NOVEL, and I think valuable, expedient came to my rescue as a substitute for Heurtelouping when leeches became scarce during the war. In acute and sub-acute glaucoma and other congestions 
and inflammations of the eyes, I have proved that the abstraction of blood from the nasal mucous membrane will often afford speedy relief; while as a preliminary to operations for glaucoma and cataract in haemorrhagic or dangerously plethoric individuals, it is of decided value. Sometimes patients with ophthalmic haemorrhage present also the well-known symptoms which threaten cerebral apoplexy, and then. I would advise, and, indeed, have practised, artificial epistaxis, as likely to avert a stroke. Another field of usefulness may be found in the so-called vicarious haemorrhages of the menopause.

Spontaneous nose-bleeding seems to be Nature's favourite mode of relieving congestions of the head, and can we do better than follow her example, and draw blood from the locality she indicates? Such was the consideration which led me to try it. It often happens that persons who have been liable to recurrent epistaxis for years, experience discomfort in the eyes, with the feeling of fulness, after the cessation of the habit. Indeed, we have no doubt all seen vitreous opacities or retinal haemorrhages in some cases of this kind. I well remember, many years ago, receiving the embarrassing petition from a beautiful lady, that I should give her a punch on the nose to make it bleed, as her eyes, she said, were always so much better after attacks of recurrent nose-bleeding, which had now ceased. Unfortunately, at that time I had not conceived this idea of tapping the nasal mucous membrane, and she had to be.content with the usual Heurteloup from the temple, as I could not bring myself to her proposition!

It only remains to mention how to effect the little procedure. At first I liked to see what I was doing, and passed a narrow tubular cool electrical lamp into the opposite nostril to the one chosen for attack. This turned the nose into a kind of lantern, the cartilaginous septum being semi-transparent, and the best spot for the incision of the mucous membrane could be selected with ease. I soon found it unnecessary, however, to take this trouble, unless the yield of blood without it proved too small. I simply now pass an extremely small keratome up the nostril, keeping its back against the septum, and then, when high enough up, a sudden turn of the wrist does all that is required. I* can thoroughly recommend this mode of depletion, and besides its use in suitable cases in ophthalmology, I should not be surprised if physicians might also with advantage borrow the idea for some congestive conditions of the head, especially during the crises of fevers, and invariably, I would suggest, after cerebral concussion. A sterilized penknife is nearly always obtainable in the absence of anything better. 